\title{
NONLINEAR SYMMETRIC BENDING OF CIRCULAR ELASTIC PLATES
}

\author{
B. P. GARFOOT \\ (Received 28 March 1973; revised 27 July 1973) \\ Communicated by V. G. Hart
}

In recent years, there has been considerable interest in the refining of thin plate theories. In this paper, the method of matching asymptotic expansions is used to obtain one such refinement which is believed to be an improvement on several previous results. Previous authors (Habip (1967), Widera (1969)) attempted such refinements within the framework of a partially nonlinear theory of elasticity whereas in the present work all terms neglected by these authors have been retained.

The forms of the solutions for the displacements and stresses in the interior of the plate are given in equations (3.7) to (3.12) while those for the boundary layer are given in (4.40) to (4.45). On comparing both sets of solutions it will be observed that the order of magnitude of the stresses increases near the edge of the plate. This effect is due to the occurrence of a boundary layer depending on a dimensionless thickness parameter $\varepsilon$ defined in $\$ 1$. Schematic diagrams illustrating the change in the order of the stresses appear at the end of the paper.

The assumptions made on the magnitude of the deformation are given in $\$ 2$.

\section{Formulation of the problem}

We consider a thin isotropic ideally elastic circular plate of radius $R_{0}$, thickness $2 \mathrm{H}$, with the origin of the Langrangian coordinates at the centre on the middle surface. The plate is initially flat. We shall use cylindrical polar coordinates $R, \Theta, Z$. Thus

$$
0 \leqq R \leqq R_{0},-H \leqq Z \leqq H, 0 \leqq \Theta \leqq 2 \pi
$$

for the plate.

In deformations symmetrical about the origin, the two non-zero displacements are independent of $\Theta$. Thus the material point with coordinates $R, \Theta, Z$ before the deformation has coordinates $R+U(R, Z), \Theta, Z+W(R, Z)$ after the defor- 
mation where $U(R, Z)$ is the radial displacement and $W(R, Z)$ is the lateral displacement.

We define dimensionless independent variables $x$ and $\zeta$ by setting

$$
R=x R_{0} \text { and } Z=\zeta H .
$$

Thus

$$
0 \leqq x \leqq 1 \text { and }-1 \leqq \zeta \leqq 1
$$

Dimensionless stress and displacement components $T^{K L}, u(x, \zeta)$ and $w(x, \zeta)$ are defined by

$$
U=R_{0} u, \quad W=R_{0} w,
$$

$E T^{11}=\stackrel{*}{T}{ }^{11}, E T^{13}=\stackrel{*}{T^{13}}, E T^{33}=\stackrel{*}{T}^{33}, E T^{22}=R^{2} \stackrel{*}{T}^{22}$, where $\stackrel{*}{T}^{K L}$ is the Piola stress tensor, (Eringen (1962)) and $E$ is Young's Modulus for the material.

The stresses $\stackrel{*}{T}^{12}$ and $\stackrel{*}{T}^{23}$ are zero since we are considering symmetrical bending.

A thickness to plate 'width' ratio $\varepsilon$ may be defined as

$$
\varepsilon=\frac{H}{R_{0}} .
$$

Using these dimensionless variables, the equilibrium equations, straindisplacement relations and strain invariants are as follows:

The non-dimensional forms of equilibrium equations are:

$$
\varepsilon\left(1+u,_{x}\right) T_{, x}^{11}+\varepsilon u,,_{x x} T^{11}+\left(1+u,,_{x}\right) T_{\zeta}^{13}+2 u,_{x \zeta} T^{13}+u,_{\zeta} T_{, x}^{13}+\varepsilon^{-1} u,,_{\zeta} T_{\zeta}^{33}
$$

and

$$
+\varepsilon^{-1} u_{\zeta \zeta} T^{33}-\varepsilon x^{-1}\left(1+x^{-1} u\right) T^{22}+\varepsilon x^{-1}\left(1+u,{ }_{x}\right) T^{11}+x^{-1} u_{\zeta} T^{13}=0
$$

$$
\begin{aligned}
& \varepsilon w,{ }_{x} T_{x}^{11}+\varepsilon w,_{x x} T^{11}+\varepsilon\left(1+\varepsilon^{-1} w, \zeta\right),_{x}^{13}+2 w,_{x \zeta} T^{13}+w_{, x} T_{\zeta}^{13}+\left(1+\varepsilon^{-1} w, \zeta\right) T_{\zeta \zeta}^{33} \\
& +\varepsilon^{-1} w,{ }_{\zeta \zeta} T^{33}+\varepsilon x^{-1} w,_{x} T^{11}+\varepsilon x^{-1}\left(1+\varepsilon^{-1} w, \zeta\right) T^{13}=0 .
\end{aligned}
$$

The strain tensor components are

$$
\begin{aligned}
E_{11} & =u,_{x}+\frac{1}{2}\left(u,_{x}\right)^{2}+\frac{1}{2}\left(w \cdot_{x}\right)^{2}, \\
R^{-2} E_{22} & =\frac{u}{x}+\frac{1}{2}\left(\frac{u}{x}\right)^{2}, \\
E_{33} & =\left(\frac{w, \zeta}{\varepsilon}\right)+\frac{1}{2}\left(\frac{u, \zeta}{\varepsilon}\right)^{2}+\frac{1}{2}\left(\frac{w, \zeta}{\varepsilon}\right)^{2}, \\
E_{13} & =\frac{1}{2 \varepsilon} u,_{\zeta}+\frac{1}{2} w,_{x}+\frac{1}{2 \varepsilon} u,_{x} u,_{\zeta}+\frac{1}{2 \varepsilon} w, w_{x}, \\
E_{23} & =E_{12}=0 .
\end{aligned}
$$


The strain invariants $I_{E}, I I_{E}$ and $I I I_{E}$ are given by

$$
\begin{aligned}
& I_{E}=E_{11}+\frac{1}{R^{2}} E_{22}+E_{33}, \\
& I I_{E}=E_{11} E_{33}-E_{13}^{2}+\frac{1}{R^{2}} E_{11} E_{22}+\frac{1}{R^{2}} E_{22} E_{33}, \\
& I I I_{E}=\frac{1}{R^{2}}\left(E_{11} E_{22} E_{33}-E_{22} E_{13}^{2}\right) .
\end{aligned}
$$

The comma is used in the subscripts to denote partial differentiation with respect to the variables following it.

The strain energy function $\Sigma$, from which the relations between the stresses and the strains are derived is assumed to be a single-valued analytic function or the Lagrangian coordinates and the strain invariants (as in Eringen (1962)). The natural or initial state of the body is stress free.

Thus

$$
\begin{gathered}
\Sigma=\frac{1}{2}\left(\lambda_{E}+2 \mu_{E}\right) I_{E}^{2}-2 \mu_{E} I I_{E}+l_{E} I_{E}^{3}+m_{E} I_{E} I I_{E}+n_{E} I I I_{E}+h_{E} I_{E}^{4}+k_{E} I I_{E}^{2} \\
+p_{E} I_{E} I I I_{E}+{ }_{q E} I_{E}^{2} I_{E}+\cdots
\end{gathered}
$$

Dimensionless constants $v_{1}, v_{2}, \cdots$ may be defined by $m_{E}=E v_{1}, n_{E}=E v_{2}$, $l_{E}=E v_{3}, p_{E}=E v_{4}, q_{E}=E v_{5}, h_{E}=E v_{6}, k_{E}=E v_{7}, \cdots$ where Young's Modulus $E$ and Poisson's ration $v$ are given by the relations

$$
2(1+v) \mu_{E}=E \text { and }(1+v)(1-2 v) \lambda_{E}=v E .
$$

It can be shown that the stresses $T^{K L}$ are given by

$$
\begin{aligned}
T^{11}=\frac{v I_{E}}{(1+v)(1-2 v)}+\frac{E_{11}}{1+v}+ & \left(v_{1}+3 v_{3}\right) I_{E}^{2}+v_{2}\left(E_{11}^{2}+E_{13}^{2}\right) \\
& +\left(v_{1}+v_{2}\right)\left(I I_{E}-I_{E} E_{11}\right)+\cdots
\end{aligned}
$$

$$
\begin{aligned}
T^{22}=\frac{v I_{E}}{(1+v)(1-2 v)}+\frac{R^{-2} E^{22}}{1+v}+ & v_{2}\left(R^{-2} E_{22}\right)^{2}+\left(v_{1}+3 v_{3}\right) I_{E}^{2} \\
& +\left(v_{1}+v_{2}\right)\left(I I_{E}-I_{E} R^{-2} E_{22}\right)+\cdots
\end{aligned}
$$

$$
\begin{aligned}
T^{33}=\frac{v I_{E}}{(1+v)(1-2 v)}+\frac{E_{33}}{1+v}+ & v_{2}\left(E_{13}^{2}+E_{33}^{2}\right)+\left(v_{1}+3 v_{3}\right) I_{E}^{2} \\
& +\left(v_{1}+v_{2}\right)\left(I I_{E}-I_{E} E_{33}\right)+\cdots \\
T^{13}= & \frac{E_{13}}{1+v}+v_{2}\left(E_{11} E_{13}+E_{33} E_{13}\right)-\left(v_{1}+v_{2}\right) I_{E} E_{13}+\cdots \\
T^{12}= & T^{23}=0
\end{aligned}
$$


The formulation of the problem will be completed by specifying boundary conditions on the $\zeta= \pm 1$ faces and on the edge $x=1$ and by giving symmetry conditions at $x=0$.

On the $\zeta= \pm 1$ faces of the plate, the dimensionless normal stress $N_{N}$ and tangential stress $N_{T}$ are specified as follows:

$$
\begin{aligned}
& N_{T}=0 \text { on } \zeta= \pm 1
\end{aligned}
$$

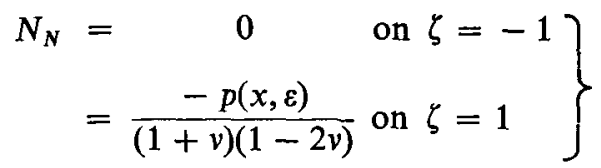

$$
\begin{aligned}
& \text { where } p(x, \varepsilon) \text { is assumed to be analytic in } \varepsilon \text {. }
\end{aligned}
$$

The expressions for $N_{T}$ and $N_{N}$ are given by

$$
N_{N}=J^{-1}\left[(1+u, x)^{2}+\left(w, x^{2}\right]^{-1}\left(1+u,_{x}+\varepsilon^{-1} w_{, \xi}\right)^{2} T^{33},\right.
$$

and

$$
\begin{aligned}
& N_{T}=\left(1+\frac{u}{x}\right)^{-1}\left\{T^{13}+\left[\left(1+u,_{x}\right)^{2}+\left(w_{, x}\right)^{2}\right]^{-1}\left[\varepsilon^{-1} u_{, \zeta}+w_{x_{x}}\right.\right. \\
& \left.\left.+\varepsilon^{-1} u, u_{, \zeta}+\varepsilon^{-1} w, x_{x} w\right] T^{33}\right\}
\end{aligned}
$$

where

$$
J=\left(1+\frac{u}{x}\right)\left(1+u,_{x}+\varepsilon^{-1} w_{, \zeta}+\varepsilon^{-1} u_{, x} w_{, \zeta}-\varepsilon^{-1} u_{,^{\prime}, x}\right) .
$$

Physically, $J$ is the ratio of the deformed to the undeformed elements of volume. Thus $J=1$ when all deforming forces are removed.

On the edge $x=1$, we consider the clamped edge conditions of radial displacement and slope specified. Thus

$$
u(1, \zeta)=\rho(\zeta, \varepsilon), \frac{w_{, x}(1, \zeta)}{1+u, x_{x}(1, \zeta)}=\psi(\zeta, \varepsilon)
$$

To completely solve the problem, $w(x, \zeta)$ must be specified at one particular point, for example:

$$
w(1,0)=\varepsilon a_{1}+\varepsilon^{2} a_{2}+\cdots
$$

Since the bending is symmetrical, conditions at $x=0$ are

$$
u(0, \zeta)=0, w_{x}(0, \zeta)=0,
$$

that is, there is no radial displacement and the slope is zero along the $Z$ (i.e. $\zeta$ ) axis. 


\section{Assumptions governing the deformation}

The following assumptions on the magnitude of the deformation will be made before attempting to solve the problem:

(i) The plate is thin, that is, $\varepsilon<<1$,

(ii) $|W|=O(H)$, thus $|w|=O(\varepsilon)$,

(iii) $|U|=O(\varepsilon H)$, thus $|u|=O\left(\varepsilon^{2}\right)$,

(iv) The slope $\frac{w_{,_{x}}}{1+u_{j_{x}}}=O(\varepsilon)$ for all $x, \zeta$, and

(v) $\Sigma$ is an analytic function of the strain invariants.

The assumptions are some of those made in the original derivation of the von Kármán equations.

The method of matched asymptotic expansions (described in Van Dyke (1964)) is used to derive the first few approximations to the displacements $u(x, \zeta)$, $w(x, \zeta)$ and the stresses $T^{K L}(x, \zeta)$.

\section{The interior problem}

In this section the first three terms for the interior expansion of each of the dimensionless dependent variables $u(x, \zeta ; \varepsilon), w(x, \zeta ; \varepsilon)$ and $T^{K L}(x, \zeta ; \varepsilon)$ are determined. The differential equations and stress-displacement relations of the large deflexion theory developed by von Kármán (1910) are found to be those of the first terms of the interior expansions. The boundary conditions on the interior variables at the edge of the plate are presented in $\S 4$ of this paper.

3.1. Asymptotic form for the solutions $u(x, \zeta ; \varepsilon), w(x, \zeta ; \varepsilon)$ and $T^{K L}(x, \zeta ; \varepsilon)$

Assumptions (ii) and (iii) of $\$ 2$ suggest that we assume interior expansions of the form

$$
\begin{aligned}
& w(x, \zeta ; \varepsilon)=\varepsilon w_{1}(x, \zeta)+\varepsilon^{2} w_{2}(x, \zeta)+\cdots \\
& u(x, \zeta ; \varepsilon)=\varepsilon^{2} u_{2}(x, \zeta)+\varepsilon^{3} u_{3}(x, \zeta)+\cdots
\end{aligned}
$$

Substituting (3.1) and (3.2) into equations (1.3) to (1.11) shows that the $T^{K L}$ will have expansions of the form

$$
\begin{aligned}
T^{K L} & =T_{0}^{K L}(x, \zeta)+\varepsilon T_{1}^{K L}(x, \zeta)+\cdots \text { for } K=L, \text { and } \\
T^{13} & =\varepsilon T_{1}^{13}(x, \zeta)+\varepsilon^{2} T_{2}^{13}(x, \zeta)+\cdots
\end{aligned}
$$

The quantity $J$ also has the expansion

$$
J=1+w_{1, \zeta}+\varepsilon w_{2, \zeta}+\cdots
$$


To find equations for the coefficients of $\varepsilon^{n}(n=0,1,2, \cdots)$ in (3.1) to (3.4) we simply substitute these expressions into the equilibrium equations (1.1 and (1.2) and apply the boundary conditions given in (1.12) and (1.13). The solving of this system of equations forms the interior problem.

It is also assumed that the function $p(x, \varepsilon)$ has the expansion

$$
p(x, \varepsilon)=p_{0}(x)+\varepsilon p_{1}(x)+\cdots
$$

\subsection{Asymptotic Solution for the Interior of the Plate}

The substituting of (3.1) to (3.5) into the equilibrium equations (1.1) to (1.2) and then equating the coefficients of $\varepsilon^{n}(n=0,1,2, \cdots)$ in turn to zero enables one to determine the $\zeta$ dependence of the interior expansions (3.1) to (3.5). It turns out that the functions $w_{\tau}(x, \zeta), u_{\tau+1}(x, \zeta), T_{\tau}^{K L}(x, \zeta)$ and $T_{\tau}^{13}(x, \zeta)$ for $\tau=1,2,3, \cdots$ are polynomials in $\zeta$. The coefficients of each power of $\zeta$ in these polynomials are functions of $x$. Application of the boundary conditions given in (1.12) and (1.13) enables one to derive differential equations for and relations between these functions of $x$ (see equations (3.14) to (3.19), (3.21) to (3.25) of this section).

This method will be illustrated by determining the form of $w_{1}(x, \zeta)$ and the stresses $T_{0}^{K L}$ in detail.

Substituting (3.1) to (3.5) into (1.2) and equating the coefficient of $\varepsilon^{0}$ to zero gives the equation

$$
\frac{\partial}{\partial \zeta}\left[\left(1+w_{1, \zeta}\right) T_{0}^{33}\right]=0
$$

Integration of this shows that

$$
\left(1+w_{1}, \zeta\right) T_{0}^{33}=K_{0}(x) .
$$

Expanding $N_{N}$ (given in (1.14)) in powers of $\varepsilon$, we obtain

$$
N_{N}=K_{0}(x)+O(\varepsilon) .
$$

Applying the boundary conditions given by equation (1.13), it follows that

$$
\begin{aligned}
& K_{0}(x)=\frac{-p_{0}(x)}{(1+v)(1-2 v)}(\text { using }(3.6) \text { on } \zeta=1, \text { and } \\
& K_{0}(x)=0 \text { on } \zeta=-1 .
\end{aligned}
$$

Thus

$$
\begin{aligned}
p_{0}(x) & =0, \\
\left(1+w_{1,5}\right) T_{0}^{33} & =0 .
\end{aligned}
$$

If we examine (1.3) to (1.10) closely, we may show that, due to the different orders in $\varepsilon$ of $w(x, \zeta ; \varepsilon)$ and $u(x, \zeta ; \varepsilon)$, 


$$
T_{0}^{33}=w_{1, \zeta} f\left(w_{1, \zeta}\right) ; T_{0}^{11}=T_{0}^{22}=w_{1, \zeta}\left[f\left(w_{1, \zeta}\right)-g\left(w_{1, \zeta}\right)\right]
$$

where $f\left(w_{1, \zeta}\right)$ and $g\left(w_{1, \zeta}\right)$ are polynomials in $w_{1, \zeta}$ with constants coefficients and infinite degree.

Thus

$$
\left(1+w_{1, \zeta}\right) w_{1, \zeta} f\left(w_{1, \zeta}\right)=0 .
$$

Therefore $w_{1, \zeta}$ is constant and may be $-1,0$ or the solution of $f\left(w_{1, \zeta}\right)=0$. Since $J=1$ for all $\varepsilon \geqq 0$ when there is no deformation, the only acceptable solution is that $w_{1, \zeta} \equiv 0$. Hence

and

$$
w_{1}(x, \zeta)=W_{1}(x)
$$

$$
T_{0}^{K L}(x, \zeta)=0
$$
obtain

By continuing the process explained at the beginning of this section, we

$$
\begin{aligned}
w(x, \zeta ; \varepsilon)=\varepsilon W_{1}(x)+ & \varepsilon^{2} W_{2}(x)+\varepsilon^{3}\left\{\frac{v \zeta^{2}}{2(1-v)} \nabla^{2} W_{1}\right. \\
& \left.+W_{3}(x)-\frac{\zeta}{1-v}\left[\frac{1}{2}\left(W_{1}^{\prime}\right)^{2}+\frac{v}{x} \frac{d}{d x}\left(x b_{2}\right)\right]\right\}+\cdots
\end{aligned}
$$

$$
\begin{aligned}
& u(x, \zeta ; \varepsilon)= \varepsilon^{2}\left[b_{2}(x)-W_{1}^{\prime}(x) \zeta\right]+\varepsilon^{3}\left[b_{3}(x)-W_{2}^{\prime}(x) \zeta\right]+\varepsilon^{4}\left\{b_{4}(x)\right. \\
&-\zeta\left[W_{3}^{\prime}(x)+\frac{1}{1-v} \frac{d}{d x}\left(\nabla^{2} W_{1}\right)-\frac{W_{1}^{\prime}}{1-v}\left\{b_{2}^{\prime}+\frac{v b_{2}}{x}+\frac{1}{2}\left(W_{1}^{\prime}\right)^{2}\right\}\right] \\
&-\frac{v \zeta^{2}}{2(1-v)}\left[W_{1}^{\prime} W_{1}^{\prime \prime}+\frac{1}{2}(3-v) \frac{\left(W_{1}^{\prime}\right)^{2}}{x}\right] \\
&\left.+\frac{1}{6}\left(\frac{2-v}{1-v}\right) \zeta^{3} \frac{d}{d x}\left(\nabla^{2} W_{1}\right)\right\}+\cdots
\end{aligned}
$$

$$
\begin{aligned}
T^{11}(x, \zeta ; \varepsilon)=\frac{\varepsilon^{2}}{1-v^{2}} & \left\{\frac{1}{3 x} \Phi_{1}^{\prime}(x)-\zeta\left[W_{1}^{\prime \prime}(x)+v \frac{W_{1}^{\prime}(x)}{x}\right]\right\} \\
+ & \frac{\varepsilon^{3}}{1-v^{2}}\left\{\frac{1}{3 x} \Phi_{2}^{\prime}(x)-\zeta\left[W_{2}^{\prime \prime}(x)+v \frac{W_{2}^{\prime}(x)}{x}\right]\right\}+\cdots
\end{aligned}
$$

$$
\begin{aligned}
T^{22}(x, \zeta ; \varepsilon)= & \frac{\varepsilon^{2}}{1-v^{2}}\left\{\frac{1}{3} \Phi_{1}^{\prime \prime}(x)-\zeta\left[v W_{1}^{\prime \prime}(x)+\frac{W_{1}^{\prime}(x)}{x}\right]\right\} \\
& +\frac{\varepsilon^{3}}{1-v^{2}}\left\{\frac{1}{3} \Phi_{2}^{\prime \prime}(x)-\zeta\left[v W_{2}^{\prime \prime}(x)+\frac{W_{2}^{\prime}(x)}{x}\right]\right\}+\cdots
\end{aligned}
$$


(3.11) $\quad T^{13}(x, \zeta ; \varepsilon)=\varepsilon^{3} \frac{\left(\zeta^{2}-1\right)}{2\left(1-v^{2}\right)} \frac{d}{d x}\left(\nabla^{2} W_{1}\right)+\varepsilon^{4} \frac{\left(\zeta^{2}-1\right)}{2\left(1-v^{2}\right)} \frac{d}{d x}\left(\nabla^{2} W_{2}\right)+\cdots$

$$
\begin{aligned}
& T^{33}(x, \zeta ; \varepsilon)=\frac{\varepsilon^{4}}{1-v^{2}}\left\{\frac{1}{6}\left(\zeta-\zeta^{3}\right) \nabla^{4} W_{1}+\frac{1}{2} \zeta^{2}\left[\left(W_{1}^{\prime \prime}\right)^{2}+2 v \frac{W_{1}^{\prime} W_{1}^{\prime \prime}}{x}\right.\right. \\
& \left.+\frac{\left(W_{1}^{\prime}\right)^{2}}{x^{2}}\right]-\frac{1}{2}\left(\frac{1-v}{1-2 v}\right) \zeta p_{4}(x)-\frac{1}{2}\left[\left(W_{1}^{\prime \prime}\right)^{2}+2 v \frac{W_{1}^{\prime} W_{1}^{\prime \prime}}{x}+\frac{\left(W_{1}^{\prime}\right)^{2}}{x^{2}}\right. \\
& \left.\left.+\left(\frac{1-v}{1-2 v}\right) p_{4}(x)\right]\right\}+\frac{\varepsilon^{5}}{1-v^{2}}\left\{\frac{1}{6}\left(\zeta-\zeta^{3}\right) \nabla^{4} W_{2}-\frac{1}{2}\left(\frac{1-v}{1-2 v}\right)(1+\zeta) p_{5}(x)\right. \\
& \left.+\left(\zeta^{2}-1\right)\left[W_{1}^{\prime \prime} W_{2}^{\prime \prime}+\frac{v}{x} \frac{d}{d x}\left(W_{1}^{\prime} W_{2}^{\prime}\right)+\frac{W_{1}^{\prime} W_{2}^{\prime}}{x^{2}}\right]\right\}+\cdots
\end{aligned}
$$

(3.13) $p(x, \varepsilon)=\varepsilon^{4} p_{4}(x)+\varepsilon^{5} p_{5}(x)+\cdots$

Higher terms in the series given in (3.7) to (3.12) are considerably more complicated than those already listed.

The functions $\Phi_{i}(x)$ are middle surface stress potentials and are defined by setting

$$
T_{i}^{11}(x, 0)=\frac{1}{1-v^{2}} \frac{\Phi_{i}^{\prime}(x)}{3 x}
$$

The method for finding the $T_{n}^{K L}$ (outlined earlier in this section) gives the results

and

$$
T_{2}^{11}(x, 0)=\frac{1}{1-v^{2}}\left[b_{2}^{\prime}+\frac{v b_{2}}{x}+\frac{1}{2}\left(W_{1}^{\prime}\right)^{2}\right]
$$

$$
T_{3}^{11}(x, 0)=\frac{1}{1-v^{2}}\left[b_{3}^{\prime}+\frac{v b_{3}}{x}+W_{1}^{\prime} W_{2}^{\prime}\right]
$$

Thus we have equations defining the middle surface stress potentials.

The functions $b_{i}(x)$ give the radial displacements of points on the middle surface and appear in the same way as the lateral displacements $W_{i}(x)$.

The differential equations for $W_{1}(x), \Phi_{1}^{\prime}(x)$ and $b_{2}(x)$ are [after considerable work]:

$$
\nabla^{4} W_{1}=-\frac{3}{2}\left(\frac{1-v}{1-2 v}\right) p_{4}(x)+\frac{1}{x} \frac{d}{d x}\left(W_{1}^{\prime} \Phi_{1}^{\prime}\right)
$$




$$
\frac{d}{d x}\left(\nabla^{2} \Phi_{1}\right)=-\frac{3}{2}\left(1-v^{2}\right) \frac{\left(W_{1}^{\prime}\right)^{2}}{x},
$$

and

$$
\Omega\left(b_{2}\right)=-W_{1}^{\prime} W_{1}^{\prime \prime}-\frac{(1-v)}{2} \frac{\left(W_{1}^{\prime}\right)^{2}}{x},
$$

where the differential operator $\Omega$ is defined by

$$
\mathbf{\Omega}\left(b_{2}\right)=\frac{d}{d x}\left\{\frac{1}{x} \frac{d}{d x}\left(x b_{2}\right)\right\},
$$

and $\nabla^{2}$ is the Laplacian in cylindrical polar coordinates.

Instead of (3.15) we may use the defining equation of $\Phi_{1}^{\prime}(x)$, that is

$$
\Phi_{1}^{\prime}(x)=3\left[x b_{2}^{\prime}+v b_{2}+\frac{1}{2} x\left(W_{1}^{\prime}\right)^{2}\right],
$$

and instead of (3.16) we may use

$$
3\left(1-v^{2}\right) b_{2}(x)=x \Phi_{1}^{\prime \prime}(x)-v \Phi_{1}^{\prime}(x) .
$$

The boundary conditions on $W_{1}(x), \Phi_{1}^{\prime}(x)$ and $b_{2}(x)$ at $x=0$ are, using equation (1.18),

$$
b_{2}(0)=W_{1}^{\prime}(0)=\Phi_{1}^{\prime}(0)=\left[\frac{d}{d x}\left(\nabla^{2} W_{1}\right)\right]_{x=0}=0 .
$$

Further, $W_{1}(0)$ and $\Phi_{1}^{\prime \prime}(0)$ are finite.

It should be noted that equations (3.14) and (3.15) are the celebrated von Kármán equations. The solution of these equations gives the first approximation to the interior problem.

The differential equations for $W_{2}(x), \Phi_{2}^{\prime}(x)$ and $b_{3}(x)$ are:

$$
\begin{gathered}
\nabla^{4} W_{2}=-\frac{3}{2}\left(\frac{1-v}{1-2 v}\right) p_{5}(x)+\frac{1}{x} \frac{d}{d x}\left(W_{1}^{\prime} \Phi_{2}^{\prime}+W_{2}^{\prime} \Phi_{1}^{\prime}\right), \\
\frac{d}{d x}\left(\nabla^{2} \Phi_{2}\right)=-3\left(1-v^{2}\right) \frac{W_{1}^{\prime} W_{2}^{\prime}}{x},
\end{gathered}
$$

and

$$
\Omega\left(b_{3}\right)=-\frac{d}{d x}\left(W_{1}^{\prime} W_{2}^{\prime}\right)-(1-v) \frac{W_{1}^{\prime} W_{2}^{\prime}}{x} .
$$

Notice that these equations are linear in $W_{2}(x), \Phi_{2}^{\prime}(x)$ and $b_{3}(x)$ unlike the von Kármán equations.

Instead of equations (3.22) and (3.23) we may use

$$
\Phi_{2}^{\prime}(x)=3\left[x b_{3}^{\prime}+v b_{3}+x W_{1}^{\prime} W_{2}^{\prime}\right] \text {, }
$$

and 


$$
3\left(1-v^{2}\right) b_{3}(x)=x \Phi_{2}^{\prime \prime}(x)-v \Phi_{2}^{\prime}(x) .
$$

The boundary conditions on $W_{2}(x), \Phi_{2}^{\prime}(x)$ and $b_{3}(x)$ at $x=0$ are

$$
b_{3}(0)=W_{2}^{\prime}(0)=\Phi_{2}^{\prime}(0)=\left[\frac{d}{d x}\left(\nabla^{2} W_{2}\right)\right]_{x=0}=0 .
$$

Further, $W_{2}(0)$ and $\Phi_{2}^{\prime \prime}(0)$ are finite.

The solution of equations (3.21) to (3.23) gives the second approximation to the interior problem.

\subsection{Discussion}

Previous attempts (Habip (1967) and Widera (1969)) to obtain an asymptotic theory for the moderately large deflexion of a transversely isotropic plate and an anisotropic plate by assuming partially nonlinear expressions for the $E_{K L}$ are in error.

Both authors assumed that

$$
E_{33} \simeq \frac{1}{\varepsilon} w, \zeta,
$$

thus neglecting terms like $\frac{1}{2}\left(\frac{u, \zeta}{\varepsilon}\right)^{2}$ in the expression for $E_{33}$.

This assumptions is incorrect since calculations show that the order of magnitude of these nonlinear terms is the same as that of the term $(1 / \varepsilon) w, \zeta^{*}$. A similar error was made in their expression for terms corresponding to $E_{13}$. It is clear that the results in these two papers will be incorrect.

The approximations obtained here will not, in general, be valid near the circular edge of the plate due to the occurrence of a boundary layer. Further, boundary conditions on the functions $W_{1}(x), W_{2}(x), b_{2}(x), b_{3}(x), \Phi_{1}^{\prime}(x)$ and $\Phi_{2}^{\prime}(x)$ at $x=1$ can only be obtained by studying the boundary layer problem. This is the topic in $\S 4$ of this paper.

At this point, it is interesting to note that the boundary layer considered in this work depends upon $\varepsilon$, while the solution of the von Kármán equations often involves the consideration of a boundary layer due to a large load parameter $L^{* *}$. This leads to a two parameter expansion for $(\partial w / \partial x)$ of the form

$$
\begin{aligned}
\frac{\partial w}{\partial x}=\varepsilon & \varepsilon L^{1 / 3} W_{11}^{\prime}+\varepsilon W_{12}^{\prime}+\varepsilon L^{-1 / 3} W_{13}^{\prime}+\cdots \\
& +\varepsilon^{2} L^{1 / 3} W_{21}^{\prime}+\varepsilon^{2} W_{22}^{\prime}+\varepsilon^{2} L^{-1 / 3} W_{23}^{\prime}+\cdots
\end{aligned}
$$

* The calculations are based on the solutions given in equations (3.7) and (3.8); thus $w, \xi=0\left(\varepsilon^{2}\right)$. ** Hart and Evans (1964) used a load parameter $k$ which is related to $L$ by $k=\sqrt{3\left(1-v^{2}\right)} / L^{t}$. 
where the solutions to the von Kármán equations and equations (3.21) and (3.22) may be written as

$$
W_{i}^{\prime}(x)=L^{1 / 3} W_{i 1}^{\prime}+W_{i 2}^{\prime}+L^{-1 / 3} W_{i 3}^{\prime}+\cdots, \quad i=1,2 .
$$

In terms of the given applied load, $p_{4}(x)=O(L)$; explicitly,

$$
p_{4}(x)=2 L(1+v)(1-2 v) q_{4}(x)
$$

where $q_{4}(x)=O(1)$ compared with $L$.

\section{The boundary layer problem.}

The results of $\S 3$ were obtained without any reference to the boundary conditions on the edge $x=1$. Since the functional form of the interior expansions has been determined, it is clear that these expansions cannot, in general, satisfy the clamped edge boundary conditions given in $\S 1$ since $\rho(\zeta, \varepsilon)$ and $\psi(\zeta, \varepsilon)$ are essentially arbitrary functions of $\zeta$. Thus the interior expansions only represent the solution away from the boundary.

Unlike the variational approach of Reissner (1963), which attempted to determine boundary conditions on the interior solutions, we are concerned with the effects of the boundary layer solutions on the boundary conditions of the interior solutions. Thus we use a boundary layer approach similar to that carried out by Reiss (1962), Reiss and Locke (1961), Friedrichs and Dressler (1961), Kolos (1964) and Gol'Denveizer and Kolos (1965), for the linear theory. Since the present work is highly nonlinear in character, the calculations are considerably more complicated than the work by these authors. Thus most calculations will be omitted and only sufficient work will be presented to enable the determination of the first two nonzero terms in the boundary layer expansions of $u(x, \zeta, \varepsilon)$, $w(x, \zeta, \varepsilon)$ and the $T^{K L}(x, \zeta, \varepsilon)$.

The final form of the boundary layer solutions appear in equations (4.40) to (4.45).

\subsection{Formulation of the boundary layer problem}

Similar to Reiss (1962), we 'stretch' the coordinate normal to the edge by defining a new coordinate $\eta$ by

$$
\eta=\frac{1-x}{\varepsilon}
$$

This stretching (4.1) implies that the boundary layer effects penetrate a 'distance' of order of magnitude $\varepsilon$ into the plate from the edge. The non-dimensional forms of the equilibrium equations (1.1) and (1.2) in terms of $\eta$ and $\zeta$ are: 
(4.2)

$$
\begin{aligned}
- & \left(1-\varepsilon^{-1} u_{, \eta}\right) T_{\eta}^{11}+\varepsilon^{-1} u_{, \eta \eta} T^{11}+\left(1-\varepsilon^{-1} u_{\eta}\right) T_{\zeta \zeta}^{13} \\
& -2 \varepsilon^{-1} u_{\eta \zeta} T^{13}-\varepsilon^{-1} u_{, \zeta} T_{\eta \eta}^{13}+\varepsilon^{-1} u_{\zeta \zeta} T_{\zeta}^{33} \\
& +\varepsilon^{-1} u_{\zeta \zeta} T^{33}+x^{-1} u_{, \zeta} T^{13}+\varepsilon x^{-1}\left(1-\varepsilon^{-1} u_{, \eta}\right) T^{11} \\
& -\varepsilon x^{-1}\left(1+x^{-1} u\right) T^{22}=0 .
\end{aligned}
$$

and

$$
\begin{aligned}
& \varepsilon^{-1} w_{, \eta} T_{\eta \eta}^{11}+\varepsilon^{-1} w_{\eta \eta} T^{11}-\left(1+\varepsilon^{-1} w_{, \zeta}\right) T_{\eta \eta}^{13} \\
& -2 \varepsilon^{-1} w_{, \eta \zeta} T^{13}-\varepsilon^{-1} w_{, \eta} T_{\zeta}^{13}+\left(1+\varepsilon^{-1} w_{\zeta \zeta}\right) T_{, \zeta}^{33} \\
& +\varepsilon^{-1} w_{, \zeta \zeta} T^{33}+\varepsilon x^{-1}\left(1+\varepsilon^{-1} w, \zeta\right) T^{13} \\
& -x^{-1} w_{, \eta} T^{11}=0 .
\end{aligned}
$$

The strain tensor components may be rewritten as

$$
\begin{aligned}
E_{11} & =-\frac{u, \eta}{\varepsilon}+\frac{1}{2}\left(\frac{u_{, \eta}}{\varepsilon}\right)^{2}+\frac{1}{2}\left(\frac{w, \eta}{\varepsilon}\right)^{2} \\
R^{-2} E_{22} & =\frac{u}{x}+\frac{1}{2}\left(\frac{u}{x}\right)^{2} \\
E_{33} & =\frac{w_{, \zeta}}{\varepsilon}+\frac{1}{2}\left(\frac{u, \zeta}{\varepsilon}\right)^{2}+\frac{1}{2}\left(\frac{w_{, \zeta}}{\varepsilon}\right)^{2} \\
E_{13} & =\frac{1}{2 \varepsilon} u_{, \zeta}-\frac{1}{2 \varepsilon} w_{, \eta}-\frac{1}{2 \varepsilon^{2}} u_{, \eta} u_{, \zeta}-\frac{1}{2 \varepsilon^{2}} w_{, \eta} w_{, \zeta}
\end{aligned}
$$

and

$$
E_{12}=E_{23}=0 \text {. }
$$

The strain invariants $I_{E}, I I_{E}$ and $I I I_{E}$ are given by equations (1.7) in $\$ 1$.

The equations for the non-dimensional stresses $T^{K L}$ are given by (1.8) to (1.11) in $\S 1$.

The expression for the slope at the edge transforms to

$$
\frac{-\varepsilon^{-1} w_{\eta}(1, \zeta)}{1-\varepsilon^{-1} u, \eta(1, \zeta)}
$$

The quantity $J$ becomes

$$
J=\left(1+\frac{u}{x}\right)\left(1-\frac{u_{, \eta}}{\varepsilon}+\frac{w, \zeta}{\varepsilon}-\frac{u_{, \eta} w_{, \zeta}}{\varepsilon^{2}}+\frac{u_{, \zeta} w, \eta}{\varepsilon^{2}}\right)
$$

On the faces $\zeta= \pm 1$ we have the conditions

(4.10) $N_{N}=J^{-1}\left[\left(1-\varepsilon^{-1} u_{\eta \eta}\right)^{2}+\left(\varepsilon^{-1} w_{\eta \eta}\right)^{2}\right]^{-1} \times\left(1-\varepsilon^{-1} u, \eta+\varepsilon^{-1} w, \xi\right)^{2} T^{33}$ 


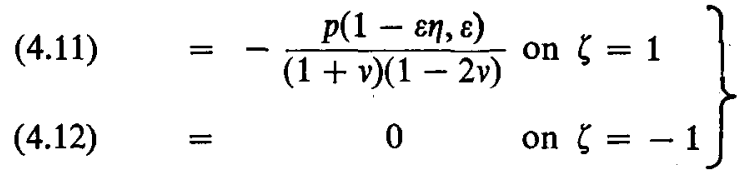

and

(4.13) $N_{T}=\left(1+\frac{u}{x}\right)^{-1}\left\{T^{13}-\left[\left(1-\varepsilon^{-1} u_{, \eta}\right)^{2}+\left(\varepsilon^{-1} w_{, \eta}\right)^{2}\right]^{-1} \times\left(-\varepsilon^{-1} u, \zeta\right.\right.$

$$
\left.\left.+\varepsilon^{-1} w, \eta+\varepsilon^{-2} u, \eta, \zeta+\varepsilon^{-2} w, w_{, \zeta}\right) T^{33}\right\}
$$

(4.14) $=0$ on $\zeta= \pm 1$.

On the edge $\eta=0$ (i.e. $x=1$ ), the boundary conditions applicable are (1.18) and

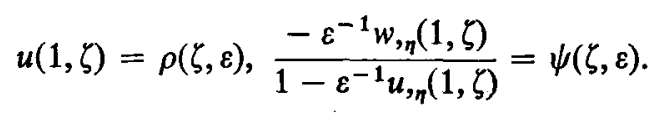

As will be seen later in equations such as $(4.48), \rho(\zeta, \varepsilon)$ and $\psi(\zeta, \varepsilon)$ cannot be arbitrarily prescribed.

4.2 Asymptotic form of the solutions $u(x, \zeta ; \varepsilon), w(x, \zeta ; \varepsilon)$ and $T^{K L}(x, \zeta ; \varepsilon)$.

We define dimensionless boundary layer displacements and stresses $\hat{u}, \hat{w}$ and $\hat{T}^{K L}$ by

$$
\begin{aligned}
\hat{u}(\eta, \zeta ; \varepsilon) & =u(x, \zeta ; \varepsilon) \\
\hat{w}(\eta, \zeta ; \varepsilon) & =w(x, \zeta ; \varepsilon) \\
\hat{T}^{K L}(\eta, \zeta ; \varepsilon) & =T^{K L}(x, \zeta ; \varepsilon)
\end{aligned}
$$

Assumptions (ii) and (iii) of $\$ 2$ suggest that we assume boundary layer expansions of the form

$$
\hat{w}(\eta, \zeta ; \varepsilon)=\varepsilon \hat{w}_{1}(\eta, \zeta)+\varepsilon^{2} \hat{w}_{2}(\eta, \zeta)+\cdots
$$

and

$$
\hat{u}(\eta, \zeta ; \varepsilon)=\varepsilon^{2} \hat{u}_{2}(\eta, \zeta)+\varepsilon^{3} \hat{u}_{3}(\eta, \zeta)+\cdots
$$

Substituting (4.17) and (4.18) into equations (1.8) to (1.11) shows that the $\hat{T}^{\mathrm{KL}}$ have expansions of the form

$$
\hat{T}^{K L}(\eta, \zeta ; \varepsilon)=\hat{T}_{0}^{K L}(\eta, \zeta)+\varepsilon \hat{T}_{1}^{K L}(\eta, \zeta)+\cdots
$$

while the quantity $J$ has the expansion

$$
J=1+\hat{w}_{1, \zeta}+\varepsilon\left[\hat{w}_{2, \zeta}-\hat{u}_{2, \eta}+\hat{u}_{2, \zeta} \hat{w}_{1, \eta}-\hat{u}_{2 \eta} \hat{w}_{1, \zeta}\right]+\cdots
$$


Assumptions (ii) and (v) of $\$ 2$ suggest that we assume

$$
\begin{aligned}
& \rho(\zeta, \varepsilon)=\varepsilon^{2} \rho_{2}(\zeta)+\varepsilon^{3} \rho_{3}(\zeta)+\cdots \\
& \psi(\zeta, \varepsilon)=-\varepsilon \psi_{2}(\zeta)-\varepsilon^{2} \psi_{3}(\zeta)+\cdots
\end{aligned}
$$

\subsection{Matching conditions}

The basic asymptotic matching principle (Van Dyke (1964), page 90) for the present problem requires that:

the boundary layer expansion of the interior expansion

$=$ the interior expansion of the boundary layer expansion.

This principle requires that the terms of the boundary layer solutions can be written in the form:

$$
\begin{array}{ll}
\hat{u}_{n}(\eta, \zeta)=-\bar{u}_{n}(\eta, \zeta)+\sum_{r=0}^{n} \frac{(-1)^{r}}{r !} \eta^{r}\left[\frac{\partial^{r}}{\partial x^{r}}\left(u_{n-r}(x, \zeta)\right)\right]_{x=1}, & n=2,3, \cdots \\
\hat{w}_{n}(\eta, \zeta)=\bar{w}_{n}(\eta, \zeta)+\sum_{r=0}^{n} \frac{(-1)^{r}}{r !} \eta^{r}\left[\frac{\partial^{r}}{\partial x^{r}}\left(w_{n-r}(x, \zeta)\right)\right]_{x=1}, & n=1,2, \cdots
\end{array}
$$

and

$$
\hat{T}_{n}^{K L}(\eta, \zeta)= \pm t_{n}^{K L}(\eta, \zeta)+\sum_{r=0}^{n} \frac{(-1)^{r}}{r !} \eta^{r} \times\left[\frac{\partial^{r}}{\partial x^{r}}\left(T_{n-r}^{K L}(x, \zeta)\right)\right]_{\substack{x=1 \\ n=1,2,3, \cdots}},
$$

where $\bar{u}_{n}, \bar{w}_{n}$ and $t_{n}^{K L}$ decay to zero as $\eta \rightarrow \infty$, the plus sign being taken for $K=L=1,2,3$, and the minus sign taken for $\hat{T}_{n}{ }^{13}$.

The remaining terms in (4.23) to (4.25) are the interior contributions to the boundary layer solutions. The boundary layer expansion for the function $p(x, \varepsilon)$ defined by equation (3.6) has the form.

$$
p(x, \varepsilon)=\sum_{n=4}^{\infty} \varepsilon^{n} \sum_{r=0}^{n} \frac{(-\eta)^{r}}{r !}\left[\frac{d^{r} p_{n-r}}{d x^{r}}\right]_{x=1}
$$

Substituting equations (4.17) to (4.19) and (4.23) to (4.25) into equations (4.2) to (4.7) and (1.7) to (1.11), we obtain sets of equations which, together with the appropriate boundary conditions, constitutes the boundary layer problem.

\subsection{Asymptotic solution for the edge of the plate}

In a manner similar to that for finding $w_{1}(x, \zeta)$, we can show that

$$
\begin{aligned}
\hat{w}_{1}(\eta, \zeta) & =\hat{w}_{1}(\mathrm{a} \text { constant }), \\
T_{0}^{K L}(\eta, \zeta) & =0
\end{aligned}
$$

Matching conditions require that

$$
\hat{w}_{1}(\eta, \zeta)=W_{1}(1)
$$


The procedure for determining the rest of the terms in equations (4.17) to (4.19) (or equivalently (4.23) to (4.25)) is considerably more complicated. Luckily, a general procedure exists. We shall describe this in detail.

The $t_{n}^{K L}(\eta, \zeta)$ may be written as (by using (4.25), (4.19) and (4.4) to (4.7) and (1.7) to (1.11))

$$
\begin{aligned}
& t_{n-1}^{11}=\frac{1}{(1+v)(1-2 v)}\left[(1-v) \bar{u}_{n, \eta}+v \bar{w}_{n, \zeta}\right]+q_{n-1}^{11}, \\
& t_{n-1}^{13}=\frac{1}{2(1+v)}\left[\bar{u}_{n, \zeta}+\bar{w}_{n, \eta}\right]+q_{n-1}^{13}, \\
& t_{n-1}^{33}=\frac{1}{(1+v)(1-2 v)}\left[v \bar{u}_{n, \eta}+(1-v) \bar{w}_{n \cdot \zeta}\right]+q_{n-1}^{33}
\end{aligned}
$$

and

$$
t_{n-1}^{22}=\frac{v}{(1+v)(1-2 v)}\left[\bar{u}_{n, \eta}+\bar{w}_{n, \xi}\right]+q_{n-1}^{22},
$$

where $n=2,3,4, \cdots$ and the $q_{n-1}^{K L}(\eta, \zeta)$ can be found in terms of $\bar{u}_{m-1}, \bar{w}_{m-1}$ and the $t_{m-2}^{K L}$ for $m=3,4, \cdots, n$.

The differential equations satisfied by the $t_{n}^{K L}$ are

$$
t_{n, \eta}^{11}+t_{n, \zeta}^{13}=X_{n}(\eta, \zeta)
$$

and

$$
t_{n, \eta}^{13}+t_{n, \zeta}^{33}=Y_{n}(\eta, \zeta)
$$

where $X_{n}$ and $Y_{n}$ decay to zero as $\eta \rightarrow \infty$ and can be found in terms of $\bar{u}_{m}, \bar{w}_{m}$, the $t_{m-1}^{K L}, X_{m-1}, Y_{m-1}, \hat{u}_{m}, \hat{w}_{m}$ and the $\hat{T}_{m-1}^{K L}$ for $m=3,4, \cdots, n$.

Using equations (4.29), (4.30) and (4.31), equations (4.33) and (4.34) may be rewritten as

$$
2(1-v) \bar{u}_{n+1, \eta \eta}+(1-2 v) \bar{u}_{n+1, \zeta 5}+\bar{w}_{n+1, \eta \zeta}=2(1+v)(1-2 v) \bar{X}_{n}
$$

and

$$
2(1-v) \bar{w}_{n+1 . \zeta \zeta}+(1-2 v) \bar{w}_{n+1, \eta \eta}+\bar{u}_{n+1, \eta \zeta}=2(1+v)(1-2 v) \bar{Y}_{n}
$$

where

$$
X_{n}=X_{n}-q_{n, \eta}^{11}-q_{n, \zeta}^{13}
$$

and

$$
\bar{Y}_{n}=Y_{n}-q_{n, \eta}^{13}-q_{n, \zeta}^{33} .
$$

Equations (4.33) to (4.36) hold for $-1 \leqq \zeta \leqq 1,0 \leqq \eta<\infty$; this is the domain of a semi-infinite strip problem. 
Using (4.10) to (4.14), it can be shown that

$$
t_{n}^{33}(\eta, \pm 1)=t_{n}^{13}(\eta, \pm 1)=0
$$

while on $\eta=0$ (i.e. $x=1$ ), the boundary conditions are given by equations (4.15). Applying the Laplace Transform to (4.29) to (4.39), it can be shown* that necessary and sufficient conditions for the $t_{n}^{K L}, \vec{u}_{n+1}$ and $\vec{w}_{n+1}$ to decay to zero as $\eta \rightarrow \infty$ are (see Appendix in Garfoot (1970)):

(Dn. 1) $\int_{-1}^{1} t_{n}^{11}(0, \zeta) d \zeta=-\int_{-1}^{1} \int_{0}^{\infty} X_{n}(\eta, \zeta) d \eta d \zeta=A_{n 1}$

(Dn. 2) $\int_{-1}^{1} \zeta t_{n}^{11}(0, \zeta) d \zeta=-\int_{-1}^{1} \int_{0}^{\infty}\left[\zeta X_{n}-\eta Y_{n}\right] d \eta d \zeta=A_{n 2}$

(Dn. 3) $\int_{-1}^{1} t_{n}^{13}(0, \zeta) d \zeta=-\int_{-1}^{1} \int_{0}^{\infty} Y_{n} d \eta d \zeta=A_{n 3}$

(Dn. 4) $\int_{-1}^{1} \vec{u}_{n+1}(0, \zeta) d \zeta+v(1+v) \int_{-1}^{1} \zeta t_{n}^{13}(0, \zeta) d \zeta$

$$
\begin{aligned}
= & -v(1+v) \int_{-1}^{1} \int_{0}^{\infty}\left[q_{n}^{33}+\zeta Y_{n}\right] d \eta d \zeta \\
& +\left(1-v^{2}\right) \int_{-1}^{1} \int_{0}^{\infty}\left[\eta X_{n}+q_{n}^{11}\right] d \eta d \zeta=A_{n 4}
\end{aligned}
$$

(Dn. 5) $\int_{-1}^{1} \zeta \bar{u}_{n+1}(0, \zeta) d \zeta+\frac{v}{2}(1+v) \int_{-1}^{1} \zeta^{2} t_{n}^{13}(0, \zeta) d \zeta$

$$
\begin{aligned}
= & \frac{1}{2}\left(1-v^{2}\right) \int_{-1}^{1} \int_{0}^{\infty}\left[2 \eta X_{n}-\eta^{2} Y_{n}+2(\zeta-1) \eta q_{\eta, 5}^{13}\right. \\
& \left.+2 q_{n}^{11}\right] d \zeta d \eta-\frac{v}{2}(1+v) \int_{-1}^{1} \int_{0}^{\infty}\left[\zeta^{2} Y_{n}+2 \zeta q_{n}^{33}\right] d \eta d \zeta
\end{aligned}
$$

and

(Dn. 6) $\int_{-1}^{1}\left(1-\zeta^{2}\right) \bar{w}_{n+1}(0, \zeta) d \zeta+\frac{1}{3}(1+v)(2-v)$

$$
\begin{aligned}
& \times \int_{-1}^{1} \zeta^{3} t_{n}^{11}(0, \zeta) d \zeta=2(1+v) \int_{-1}^{1} \int_{0}^{\infty} \eta \bar{Y}_{n} d \eta d \zeta \\
& -\frac{1}{3}(1+v)(2-v) \int_{-1}^{1} \int_{0}^{\infty}\left[\zeta^{3} X_{n}+3 \zeta^{2} q_{n}^{13}-\eta q_{n, \zeta}^{33}\right] d \eta d \zeta
\end{aligned}
$$

* When the solutions of the transformed equations are inverted, it will be seen that these contain non-decaying contributions which are polynomials in $\mathbf{b}$ as well as decaying contributions. Conditions (Dn.1) to (Dn.6) are obtained by setting these non-decaying polynomials to zero. See Gusein-Zade (1965) for the solution of a similar problem. 


$$
\begin{gathered}
-v(1+v) \int_{-1}^{1} \int_{0}^{\infty} \eta \zeta^{2} \bar{Y}_{n} d \eta d \zeta \\
-\frac{1}{3}\left(1-v^{2}\right) \int_{-1}^{1} \int_{0}^{\infty}\left[\eta^{3} Y_{n}-3 \eta^{2} \zeta X_{n}+6 \eta \zeta q_{n}^{11}\right] d \eta d \zeta=A_{n 6}
\end{gathered}
$$

for $n=1,2,3, \cdots$.

Each boundary condition for $\bar{u}_{n}$ and $\bar{w}_{n}$ on $\eta=0$ contains at least one unknown constant due to the contribution of the interior solutions as coefficients of $\eta^{r}$ in equations (4.23) to (4.25). These constants can be written in terms of the interior solutions evaluated at $x=1$. By applying conditions (Dn. 1) to (Dn. 6), these constants may be determined, thus $\bar{u}_{n}, \bar{w}_{n}$, the $t_{n-1}^{K L}$ and boundary conditions on the interior solutions at $x=1$ may be found as in Garfoot (1970).

In obtaining the solution of (4.33) to (4.36), one must use the expressions for the $X_{n}, Y_{n}$, and the $q_{n}^{i j}$ as given in the Appendix.

After carrying out the indicated computations the following boundary layer expansions for $\hat{u}, \hat{w}$, and the $\hat{T}^{K L}$ are obtained:

$$
\begin{aligned}
\hat{w}(\eta, \zeta ; \varepsilon)= & \varepsilon W_{1}(1)+\varepsilon^{2}\left[\bar{w}_{2}(\eta, \zeta)+W_{2}(1)-\eta W_{1}^{\prime}(1)\right]+\cdots \\
u(\eta, \zeta ; \varepsilon)= & \varepsilon^{2}\left[-\bar{u}_{2}(\eta, \zeta)+b_{2}(1)-\zeta W_{1}^{\prime}(1)\right] \\
& +\varepsilon^{3}\left[-\bar{u}_{3}(\eta, \zeta)+b_{3}(1)-W_{2}^{\prime}(1) \zeta-b_{2}^{\prime}(1) \eta+W_{1}^{\prime \prime}(1) \eta \zeta\right]+\cdots \\
\hat{T}^{11}(\eta, \zeta ; \varepsilon)= & \varepsilon t_{1}^{11}+\varepsilon^{2}\left[t_{2}^{11}+T_{2}^{11}(1, \zeta)\right] \\
& +\varepsilon^{3}\left[t_{3}^{11}+T_{3}^{11}(1, \zeta)-\eta T_{2, x}^{11}(1, \zeta)\right]+\cdots \\
\hat{T}^{22}(\eta, \zeta ; \varepsilon)= & \varepsilon t_{1}^{22}+\varepsilon^{2}\left[t_{2}^{22}+T_{2}^{22}(1, \zeta)\right] \\
& +\varepsilon^{3}\left[t_{3}^{22}+T_{3}^{22}(1, \zeta)-\eta T_{2, x}^{22}(1, \zeta)\right]+\cdots \\
\hat{T}^{13}(\eta, \zeta ; \varepsilon)= & -\varepsilon t_{1}^{13}-\varepsilon^{2} t_{2}^{13}+\varepsilon^{3}\left[-t_{3}^{13}+T_{3}^{13}(1, \zeta)\right] \\
& +\varepsilon^{4}\left[-t_{4}^{13}+T_{4}^{13}(1, \zeta)-\eta T_{3, x}^{13}(1, \zeta)\right]+\cdots \\
\hat{T}^{33}(\eta, \zeta ; \varepsilon)= & \varepsilon t_{1}^{33}+\varepsilon^{2} t_{2}^{33}+\varepsilon^{3} t_{3}^{33}+\varepsilon^{4}\left[t_{4}^{33}+T_{4}^{33}(1, \zeta)\right]+\cdots
\end{aligned}
$$

Sketches illustrating the nature of the boundary layer for the stresses appear at the end of the paper.

\subsection{Boundary conditions for the clamped edge}

The decay conditions (Dn. 1) to (Dn. 6) lead to the following boundary conditions on the interior solutions: 


$$
b_{2}(1)=\frac{1}{2} \int_{-1}^{1} \rho_{2}(\zeta) d \zeta+\frac{1}{4} v \int_{-1}^{1} \zeta \rho_{2}^{\prime}(\zeta) d \zeta-\frac{1}{4} v \int_{-1}^{1} \zeta \psi_{2}(\zeta) d \zeta
$$

$$
W_{1}^{\prime}(1)=-\frac{3}{2} \int_{-1}^{1} \zeta \rho_{2}(\zeta) d \zeta-\frac{3 v}{8} \int_{-1}^{1} \zeta^{2}\left[\rho_{2}^{\prime}(\zeta)-\psi_{2}(\zeta)\right] d \zeta,
$$

and

$$
\int_{-1}^{1}\left[\rho_{2}^{\prime}(\zeta)-\psi_{2}(\zeta)\right] d \zeta=0 .
$$

Equations (4.46) to (4.48), (4.15), (4.21) and (4.22) enable $\vec{u}_{2}(0, \zeta)$ and $\vec{w}_{2, \eta}(0, \zeta)$ to be found (see (4.56)), thus $\bar{u}_{2}(\eta, \zeta)$ and $\bar{w}_{2}(\eta, \zeta)$ can be determined.

Also

$$
\begin{aligned}
b_{3}(1)= & \frac{1}{2} \int_{-1}^{1} \rho_{3}(\zeta) d \zeta+\frac{1}{4} v \int_{-1}^{1} \zeta\left[\rho_{3}^{\prime}(\zeta)-\psi_{3}(\zeta)\right] d \zeta+\frac{1}{2} A_{24} \\
& -\frac{1}{2} v(1+v) \int_{-1}^{1} \zeta q_{2}^{13}(0, \zeta) d \zeta-\frac{1}{4} v \int_{-1}^{1} \zeta\left(\bar{u}_{2, \eta} \hat{w}_{2, \eta}\right)_{\eta=0} d \zeta
\end{aligned}
$$

and

$$
\begin{aligned}
W_{2}^{\prime}(1)= & -\frac{3}{2} \int_{-1}^{1} \zeta \rho_{3}(\zeta) d \zeta-\frac{3 v}{8} \int_{-1}^{1} \zeta^{2}\left[\rho_{3}^{\prime}(\zeta)-\psi_{3}(\zeta)\right] d \zeta-\frac{3}{2} A_{25} \\
& +\frac{3}{4} v(1+v) \int_{-1}^{1} \zeta^{2} q_{2}^{13}(0, \zeta) d \zeta+\frac{3}{8} v \int_{-1}^{1} \zeta^{2}\left(\bar{u}_{2, \eta} \hat{w}_{2, \eta}\right)_{\eta=0} d \zeta
\end{aligned}
$$

$$
\begin{aligned}
& \int_{-1}^{1}\left[\rho_{3}^{\prime}(\zeta)-\psi_{3}(\zeta)\right] d \zeta=A_{23}+2(\dot{1}+v) \int_{-1}^{1} q_{2}^{13}(0, \zeta) d \zeta \\
& \quad+\int_{-1}^{1}\left(\bar{u}_{2, \eta} \hat{w}_{2, \eta}\right)_{\eta=0} d \zeta .
\end{aligned}
$$

Equations (4.49) to (4.51) enable $\bar{u}_{3}(\eta, \zeta)$ and $\bar{w}_{3}(\eta, \zeta)$ to be determined by a method similar to that for finding $\bar{u}_{2}$ and $\bar{w}_{2}$.

Using (1.18) and (4.40) we find that

$$
W_{1}(1)=a_{1} \text {. }
$$

The consistency condition on $\psi_{4}(\zeta)$ and $\rho_{4}(\zeta)$ is

(4.53) $\left[\frac{d}{d x}\left(\nabla^{2} W_{1}\right)\right]_{x=1}-\frac{W_{1}^{\prime}(1) \Phi_{1}^{\prime}(1)}{2}+\frac{3 v}{4}\left(\nabla^{2} W_{1}\right)_{x=1} \int_{-1}^{1} \zeta \psi_{2}(\zeta) d \zeta$

$$
\begin{aligned}
& +\frac{3}{4}(1-v) W_{1}^{\prime \prime}(1) \int_{-1}^{1} \zeta\left[\psi_{2}(\zeta)+\rho_{2}^{\prime}(\zeta)\right] d \zeta \\
& -\frac{3}{4}(1-v) b_{2}^{\prime}(1) \int_{-1}^{1}\left[\psi_{2}(\zeta)-\bar{u}_{2, \zeta}(0, \zeta)+\frac{v}{1-v} \bar{w}_{2, \eta}(0, \zeta)\right] d \zeta
\end{aligned}
$$


[19]

Bending of elastic plates

499

$$
\begin{aligned}
& -\frac{1}{2} \Phi_{1}^{\prime}(1) \int_{-1}^{1} \bar{w}_{2, \eta}(0, \zeta) d \zeta+\frac{3}{2}\left[W_{1}^{\prime \prime}(1)+v W_{1}^{\prime}(1)\right] \int_{-1}^{1} \zeta \bar{w}_{2, \eta}(0, \zeta) d \zeta \\
& +\frac{3}{2} v(1+v)\left(\nabla^{2} W_{1}\right)_{x=1} \int_{-1}^{1} \int_{0}^{\infty} t_{1}^{33} d \eta d \zeta \\
& -\frac{3}{2} v_{1}(1+v)^{2}\left[v\left(\nabla^{2} W_{1}\right)_{x=1}-(1-v) W_{1}^{\prime \prime}(1)\right] \int_{1}^{1} \zeta t_{1}^{33}(0, \zeta) d \zeta \\
& =\frac{3}{2}\left(1-v^{2}\right) V_{1}
\end{aligned}
$$

where

$$
\begin{aligned}
V_{1}= & \int_{-1}^{1} \int_{0}^{\infty} Y_{3}^{*}(\eta, \zeta) d \eta d \zeta+\int_{-1}^{1} q_{3}^{13 *}(0, \zeta) d \zeta \\
& +\frac{1}{2(1+v)} \int_{-1}^{1}\left[\psi_{4}(\zeta)-\rho_{4}^{\prime}(\zeta)\right] d \zeta \\
& +\frac{1}{2(1+v)} \int_{-1}^{1}\left\{\bar{w}_{2, \eta} \bar{u}_{3, \eta}-\hat{w}_{2, \eta}\left(\bar{u}_{2, \eta}\right)^{2}-W_{1}^{\prime}(1) \bar{u}_{3, \eta}-\hat{u}_{2, \eta} \hat{w}_{3, \eta}\right\}_{\eta=0} d \zeta
\end{aligned}
$$

with $Y_{3}^{*}$ and $q_{3}^{13 *}$ given by

$$
\begin{aligned}
Y_{3}^{*}(\eta, \zeta)= & Y_{3}(\eta, \zeta)+W_{1}^{\prime \prime}(1) t_{1}^{11}+\frac{v}{1-v}\left(\nabla^{2} W_{1}\right)_{x=1} t_{1}^{33}-\bar{w}_{2, \eta \eta} T_{2}^{11}(1, \zeta), \\
q_{3}^{13 *}(0, \zeta)= & q_{3}^{13}(0, \zeta)+\frac{1}{2(1+v)}\left[-W_{1}^{\prime \prime}(1) \zeta \bar{u}_{2, \zeta}(0, \zeta)\right. \\
& \left.+\frac{\nu \zeta}{1-v}\left(\nabla^{2} W_{1}\right)_{x=1} \bar{w}_{2, \eta}(0, \zeta)+b_{2}^{\prime}(1) \bar{u}_{2, \zeta}(0, \zeta)-\frac{v b_{2}^{\prime}(1)}{1-v} \bar{w}_{2, \eta}(0, \zeta)\right] \\
& +v_{1}(1+v) t_{1}^{13}(0, \zeta)\left[\frac{v \zeta}{1-v}\left(\nabla^{2} W_{1}\right)_{x=1}-\left(\frac{1-2 v}{1-v}\right) b_{2}^{\prime}(1)-W_{1}^{\prime \prime}(1) \zeta\right] .
\end{aligned}
$$

$Y_{3}^{*}$ and $q_{3}^{13 *}$ are fully known in terms of $t_{1}^{i j}, t_{2}^{i j}, \bar{u}_{2}, \bar{u}_{3}, \bar{w}_{2}$ and $\bar{w}_{3}$.

Equations (4.46) to (4.48) and (4.52) supplement equations (3.20) to complete the boundary conditions on the differential equations for $W_{1}(x), \Phi_{1}^{\prime}(x)$ and $b_{2}(x)$. The final conditions on the differential equations for $W_{2}(x), \Phi_{2}^{\prime}(x)$ and $b_{3}(x)$ are:

$$
W_{2}(1)=a_{2}-\bar{w}_{2}(0,0)
$$

$$
\begin{aligned}
& {\left[\frac{d}{d x}\left(\nabla^{2} W_{2}\right)\right]_{x=1}-\frac{1}{2}\left[W_{1}^{\prime} \Phi_{2}^{\prime}+W_{2}^{\prime} \Phi_{1}^{\prime}\right]_{x=1}} \\
& \quad+\frac{3 v}{4}\left(\nabla^{2} W_{2}\right)_{x=1} \int_{-1}^{1} \zeta \psi_{2}(\zeta) d \zeta+\frac{3}{4}(1-v) W_{2}^{\prime \prime}(1) \int_{-1}^{1} \zeta \rho_{2}^{\prime}(\zeta) d \zeta
\end{aligned}
$$

https://doi.org/10.1017/S1446788700034510 Published online by Cambridge University Press 


$$
\begin{aligned}
& -\frac{3}{4}(1-v) \int_{-1}^{1} \psi_{2}(\zeta)\left[b_{3}^{\prime}(1)-\zeta W_{2}^{\prime \prime}(1)\right] d \zeta-\frac{1}{2} \Phi_{2}^{\prime}(1) \int_{-1}^{1} \bar{w}_{2 \eta}(0, \zeta) d \zeta \\
& +\frac{3}{2}\left[W_{2}^{\prime \prime}(1)+v W_{2}^{\prime}(1)\right] \int_{-1}^{1} \zeta \bar{w}_{2, \eta}(0, \zeta) d \zeta \\
& +\frac{3 v}{2}(1+v)\left[\nabla^{2} W_{2}\right]_{x=1} \int_{-1}^{1} \int_{0}^{\infty} t_{1}^{33} d \eta d \zeta \\
& +\frac{3}{4}(1-v) b_{3}^{\prime}(1) \int_{-1}^{1}\left[\bar{u}_{2, \zeta}(0, \zeta)-\frac{v}{1-v} \bar{w}_{2, \eta}(0, \zeta)\right] d \zeta \\
& -\frac{3}{2} v_{1}(1+v)^{2}\left[v \nabla^{2} W_{2}-(1-v) W_{2}^{\prime \prime}\right]_{x=1} \int_{-1}^{1} \zeta t_{1}^{33}(0, \zeta) d \zeta=\frac{3}{2}\left(1-v^{2}\right) V_{2}
\end{aligned}
$$

where

$$
\begin{aligned}
& V_{2}=\int_{-1}^{1} \int_{0}^{\infty} Y_{4}^{*}(\eta, \zeta) d \eta d \zeta+\int_{-1}^{1} q_{4}^{13 *}(0, \zeta) d \zeta \\
& +\frac{1}{2(1+v)} \int_{-1}^{1}\left[\psi_{5}(\zeta)-\rho_{5}^{\prime}(\zeta)+\bar{u}_{2, \eta}(0, \zeta) \psi_{4}(\zeta)-\hat{u}_{3, \eta}(0, \zeta) \psi_{3}(\zeta)+\bar{u}_{4, \eta}(0, \zeta) \psi_{2}(\zeta)\right] d \zeta
\end{aligned}
$$

with $Y_{4}^{*}(\eta, \zeta)$ given by

$$
Y_{4}=Y_{4}^{*}-\bar{w}_{2, \eta \eta} T_{3}^{11}(1, \zeta)-W_{2}^{\prime \prime}(1) t_{1}^{11}-\frac{v}{1-v}\left[\nabla^{2} W_{2}\right]_{x=1} t_{1}^{33} .
$$

Equation (4.55) is a consistency condition for $\psi_{5}(\zeta)$ and $\rho_{5}(\zeta)$.

The expression for $q_{4}^{13 *}(0, \zeta)$ is quite lengthy, however, both $q_{4}^{13 *}(0, \zeta)$ and $Y_{4}^{*}$ are fully known in terms of $t_{1}^{i j}, t_{2}^{i j}, t_{3}^{i j}, \bar{u}_{2}, \bar{u}_{3}, \bar{u}_{4}, \bar{w}_{2}, \bar{w}_{3}$ and $\bar{w}_{4}$.

The boundary conditions on $\bar{u}_{2}, \bar{u}_{3}, \bar{u}_{4}, \bar{w}_{2}, \bar{w}_{3}$ and $\bar{w}_{4}$ on the edge $\eta=0$ are:

$$
\left.\begin{array}{rl}
\bar{u}_{2}(0, \zeta) & =b_{2}(1)-W_{1}^{\prime}(1) \zeta-\rho_{2}(\zeta) \\
\bar{w}_{2, \eta}(0, \zeta) & =\psi_{2}(\zeta)+W_{1}^{\prime}(1)
\end{array}\right\}
$$

with $b_{2}(1), W_{1}^{\prime}(1)$ given by (4.46) and (4.47).

$$
\left.\begin{array}{rl}
\bar{u}_{3}(0, \zeta) & =b_{3}(1)-W_{2}^{\prime}(1) \zeta-\rho_{3}(\zeta) \\
\bar{w}_{3, \eta}(0, \zeta) & =\psi_{3}(\zeta)+\left[\bar{u}_{2, \eta} \hat{w}_{2, \eta}\right]_{\eta=0}+W_{2}^{\prime}(1)
\end{array}\right\}
$$

with $b_{3}(1), W_{2}^{\prime}(1)$ given by (4.49) and (4.50).

$$
\left.\begin{array}{rl}
\bar{u}_{4}(0, \zeta) & =b_{4}(1)-\zeta W_{3}^{\prime}(1)-\rho_{4}^{*}(\zeta) \\
\bar{w}_{4, \eta}(0, \zeta) & =W_{3}^{\prime}(1)+\psi_{4}^{*}(\zeta)
\end{array}\right\}
$$


where

and

$$
\rho_{4}^{*}(\zeta)=\rho_{4}(\zeta)-u_{4}(1, \zeta)+b_{4}(1)-\zeta W_{3}^{\prime}(1)
$$

$$
\begin{aligned}
\psi_{4}^{*}(\zeta)= & \psi_{4}(\zeta)+w_{3, x}(1, \zeta)-W_{3}^{\prime}(1)-\left[\hat{w}_{2, \eta} \hat{u}_{3, \eta}\right. \\
& \left.+\hat{w}_{3, \eta} \hat{u}_{2, \eta}+\hat{w}_{2, \eta}\left(\hat{u}_{2, \eta}\right)^{2}\right]_{\eta}=0 .
\end{aligned}
$$

Both $\rho_{4}^{*}(\zeta)$ and $\psi_{4}^{*}(\zeta)$ are known in terms of $W_{1}(x), b_{2}(x), W_{2}^{\prime}(1), \bar{u}_{2}, \bar{u}_{3}, \bar{w}_{2}$ and $\bar{w}_{3}$. Thus, in principle, $\bar{u}_{4}(\eta, \zeta)$ and $\bar{w}_{4}(\eta, \zeta)$ can be determined, so enabling $Y_{4}^{*}(\eta, \zeta)$ and $q_{4}^{13 *}(0, \zeta)$ to be calculated.

\subsection{Conclusion}

At this stage we now have sufficient information available to completely determine the first two terms in each of the expansions of the interior dependent variables as given in $\S 3$, equations (3.7) to (3.12) and the first two terms for the boundary layer expansions of the dependent variables given in equations (4.17) to (4.19). It should be noted that the boundary conditions given in equations (3.20). (4.46), (4.47) and (4.52) for the first interior approximation are similar to those for a clamped edge using the von Kármán plate theory. It is only in the boundary conditions of the second approximation that the boundary layer has an effect (see (4.49), (4.50) and (4.54)). Further, the prescribed edge conditions are not completely arbitrary and must satisfy conditions such as (4.48), (4.51), (4.53) and (4.55).

Figures 1 to 3 illustrate the change in the order of magnitude of the stresses near the edge of the plate.

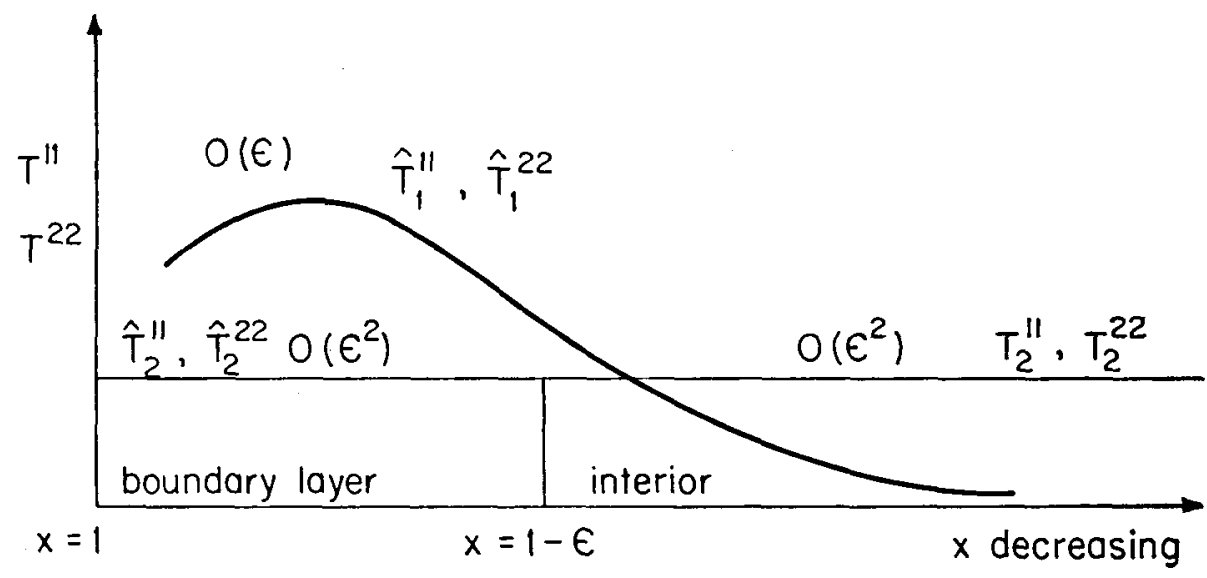

Figure 1. 


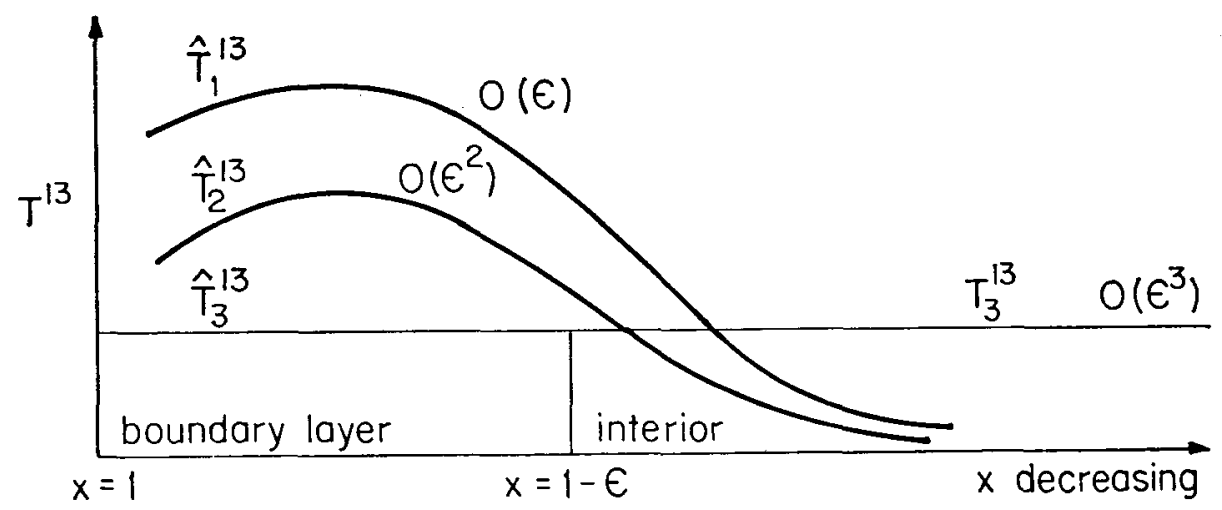

Figure 2.

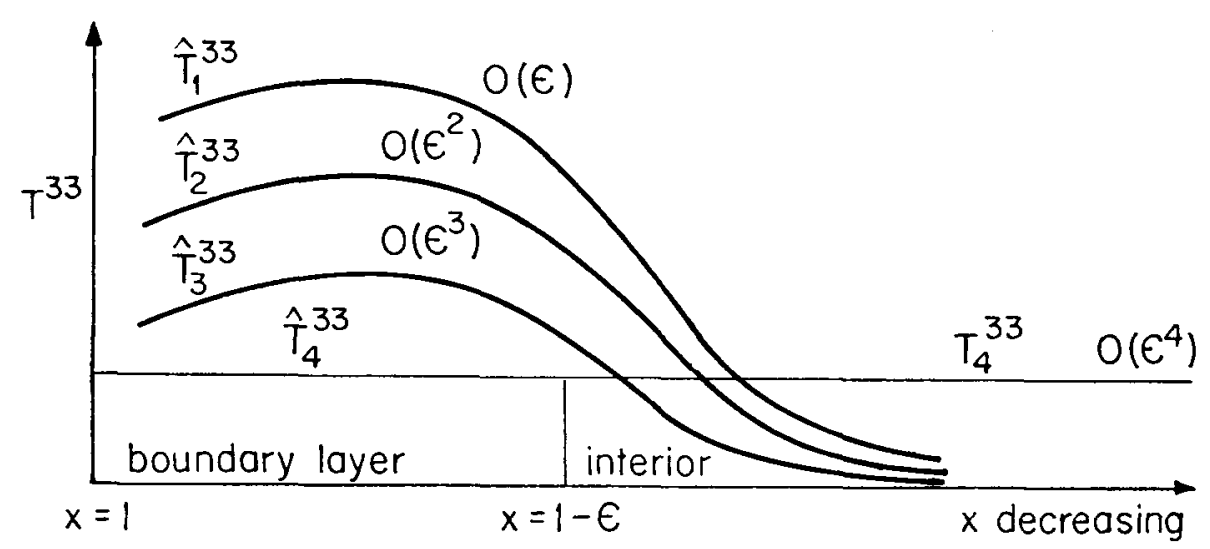

Figure 3.

Several other boundary conditions (Garfoot (1970)), such as normal and tangential stress specified on the edges, can be similarly dealt with by using the decay conditions (Dn. 1) to (Dn. 6).

\section{Acknowledgements}

The author wishes to thank Dr. V. G. Hart of the University of Queensland for his valuable cricism during - the course of this work. The gratitude of the author is also extended to the referee for his helpful comments on the work.

\section{Appendix}

On carrying out the substitutions and calculations, we find that the $X_{n}$ and $Y_{n}$ are given by 


$$
\begin{aligned}
& X_{1}(\eta, \zeta)=Y_{1}(\eta, \zeta)=0 \\
& X_{2}(\eta, \zeta)=-\bar{u}_{2, \eta \eta} t_{1}^{11}-2 \bar{u}_{2, \eta \zeta} t_{1}^{13}-\bar{u}_{2, \zeta \zeta} t_{1}^{33}+t_{1}^{11}-t_{1}^{22} \\
& Y_{2}(\eta, \zeta)=-\left\{\bar{w}_{2, \eta \eta} t_{1}^{11}+2 \bar{w}_{2, \eta \zeta} t_{1}^{13}+\bar{w}_{2, \zeta \zeta} t_{1}^{33}-t_{1}^{13}\right\} \\
& X_{3}(\eta, \zeta)=\hat{u}_{2, \zeta}\left\{Y_{2}-t_{1}^{13}\right\}+\bar{u}_{2, \eta}\left\{-X_{2}+t_{1}^{11}\right\}-\bar{u}_{3, \eta \eta} t_{1}^{11}+2 \hat{u}_{3, \eta \zeta} t_{1}^{13}-\bar{u}_{3, \zeta \zeta} t_{1}^{33} \\
& +\eta\left\{t_{1}^{11}-t_{1}^{22}\right\}-\bar{u}_{2, \eta \eta} \hat{T}_{2}^{11}+2 \bar{u}_{2, \eta \zeta} \hat{T}_{2}^{13}-\bar{u}_{2, \zeta \zeta} \hat{T}_{2}^{33}+t_{2}^{11}-t_{2}^{22} \text {, } \\
& Y_{3}(\eta, \zeta)=\hat{w}_{2, \eta}\left\{-X_{2}+t_{1}^{11}\right\}+\bar{w}_{2, \zeta}\left\{-Y_{2}+t_{1}^{13}\right\}-\hat{w}_{3, \eta \eta} t_{1}^{11}-2 \hat{w}_{3, \eta \zeta} t_{1}^{13} \\
& -\hat{w}_{3, \zeta \zeta} t_{1}^{33}+\eta t_{1}^{13}-\bar{w}_{2, \eta \eta} \hat{T}_{2}^{11}+2 \bar{w}_{2, \eta \zeta} \hat{T}_{2}^{13}-\bar{w}_{2, \zeta \zeta} \hat{T}_{2}^{33}+t_{2}^{13} \text {, } \\
& X_{4}(\eta, \zeta)=\hat{u}_{2, \zeta}\left\{Y_{3}-t_{2}^{13}-\eta t_{1}^{13}\right\}+\hat{u}_{3, \zeta}\left\{Y_{2}-t_{1}^{33}\right\}+\vec{u}_{2, \eta}\left\{-X_{3}+t_{2}^{11}+\eta t_{1}^{11}\right. \\
& \left.+T_{2}^{22}(1, \zeta)\right\}+\hat{u}_{3, \eta}\left\{X_{2}-t_{1}^{11}\right\}-\hat{u}_{2} t_{1}^{22}+t_{3}^{11}-t_{3}^{22}+\eta\left\{t_{2}^{11}-t_{2}^{22}\right\} \\
& +\eta^{2}\left\{t_{1}^{11}-t_{1}^{22}\right\}+\left\{\hat{u}_{4, \eta \eta} t_{1}^{11}+2 \hat{u}_{4, \eta \zeta} t_{1}^{13}+\hat{u}_{4, \zeta \zeta} t_{1}^{33}\right\}+\left\{-\bar{u}_{3, \eta \eta} \hat{T}_{2}^{11}\right. \\
& \left.+2 \hat{u}_{3, \eta \zeta} t_{2}^{13}-\bar{u}_{3, \zeta \zeta} t_{2}^{33}\right\}+\left\{-\bar{u}_{2, \eta \eta} \hat{T}_{3}^{11}+2 \bar{u}_{2, \eta \zeta} \hat{T}_{3}^{13}-\bar{u}_{2, \zeta \zeta} \hat{T}_{3}^{33}\right\}, \\
& Y_{4}(\eta, \zeta)=\hat{w}_{2, \eta}\left\{-X_{3}+t_{2}^{11}+\eta t_{1}^{11}\right\}+\hat{w}_{3, \eta}\left\{-X_{2}+t_{1}^{11}\right\}+\bar{w}_{2, \eta} T_{2}^{22}(1, \zeta) \\
& +t_{3}^{13}+\eta t_{2}^{13}+\eta^{2} t_{1}^{13}-W_{1}^{\prime \prime}(1) t_{2}^{11}+\bar{w}_{2, \zeta}\left\{-Y_{3}+t_{2}^{13}+\eta t_{1}^{13}\right\} \\
& +\hat{w}_{3, \zeta}\left\{-Y_{2}+t_{1}^{13}\right\}-\bar{w}_{2, \eta \eta} \hat{T}_{3}^{11}+2 \bar{w}_{2, \eta \zeta} \hat{T}_{3}^{13}-\bar{w}_{2, \zeta \zeta} \hat{T}_{3}^{33}-\bar{w}_{3, \eta \eta} \hat{T}_{2}^{11}
\end{aligned}
$$

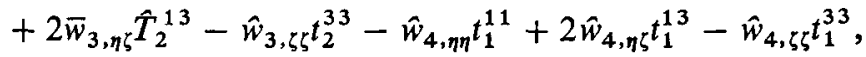

The expressions $X_{5}, Y_{5}, X_{6}, Y_{6}, \cdots$ are considerably more complicated and shall not be listed.

The $q_{n}^{i j}(\eta, \zeta)$ are given by

$$
\begin{aligned}
q_{1}^{11}= & q_{1}^{22}=q_{1}^{13}=q_{1}^{33} \equiv 0 \\
q_{2}^{11}= & \frac{1}{(1+v)(1-2 v)}\left\{\frac{(1-v)}{2}\left(\bar{u}_{2, \eta}\right)^{2}+\frac{v}{2}\left(\bar{u}_{2, \zeta}\right)^{2}+v W_{1}^{\prime}(1) \bar{u}_{2, \zeta}+\frac{v}{2}\left(\bar{w}_{2, \zeta}\right)^{2}\right. \\
& \left.+\frac{1-v}{2}\left(\bar{w}_{2, \eta}\right)^{2}-(1-v) W_{1}^{\prime}(1) \bar{w}_{2, \eta}-v \bar{u}_{2}\right\}+\left(v_{1}+3 v_{3}\right)\left(\bar{u}_{2, \eta}+\bar{w}_{2, \zeta}\right)^{2} \\
& -v_{1}\left\{\left(\bar{u}_{2, \eta}\right)^{2}+\frac{1}{4}\left(\bar{u}_{2, \zeta}+\bar{w}_{2, \eta}\right)^{2}\right\}, \\
q_{2}^{13}= & \frac{1}{2(1+v)}\left\{\bar{w}_{2, \zeta} \hat{u}_{2, \eta}-\bar{u}_{2, \eta} \hat{u}_{2, \zeta}\right\}-\frac{v_{1}}{2}\left(\bar{u}_{2, \zeta}+\bar{w}_{2, \eta}\right)\left(\bar{u}_{2, \eta}+\bar{w}_{2, \zeta}\right),
\end{aligned}
$$




$$
\begin{aligned}
q_{2}^{33}= & \frac{1}{(1+v)(1-2 v)}\left\{\frac{v}{2}\left(\bar{u}_{2, \eta}\right)^{2}+\frac{1-v}{2}\left(\bar{u}_{2, \zeta}\right)^{2}+(1-v) W_{1}^{\prime}(1) \bar{u}_{2, \zeta}\right. \\
& \left.+\frac{1}{2}(1-v)\left(\bar{w}_{2, \zeta}\right)^{2}+\frac{1}{2} v\left(\bar{w}_{2, \eta}\right)^{2}-v W_{1}^{\prime}(1) \bar{w}_{2, \eta}-v \bar{u}_{2}\right\} \\
& +\left(v_{1}+3 v_{3}\right)\left(\bar{u}_{2, \eta}+\bar{w}_{2, \zeta}\right)^{2}-v_{1}\left\{\left(\bar{u}_{2, \eta}\right)^{2}+\frac{1}{4}\left(\bar{u}_{2, \zeta}+\bar{w}_{2, \eta}\right)^{2}\right\} .
\end{aligned}
$$

The expressions for $q_{n}^{i j}, n=3,4, \cdots$ are complicated and shall not be given.

\section{References}

M. Van Dyke (1964), Perturbation Methods in Fluid Mechanics. (Academic Press, New York, 1964.)

A. C. Eringen (1962), Nonlinear Theory of Continuous Media. (McGraw-Hill Book Company, Inc., New York, 1962.)

L. M. Habip (1967), 'Moderately large deflection of asymmetrically layered elastic plate', Int. Solids Structures, 3, 207-215.

V. G. Hart and D. J. Evans (1964), 'Non-linear bending of an annular plate by transverse edge forces', J. Math. and Physics 43, 275-303.

Th. von Kármán (1910), Enzyklopädie der Mathematischen Wissenschaften. IV. 4, 1910 p. 349.

O. E. Widera (1969), 'An Asymptotic Theory for Moderately Large Deflections of Anisotropic Plates', Journal of Engineering Math. 3, 239-244.

K. O. Friedrichs and R. F. Dressler (1961), 'A Boundary Layer Theory for Elasticity Bending of Plates', Comm. on Pure and Appl. Maths. 14, 1-33.

B. P. Garfoot (1970), Ph.D. Thesis, (University of Queensland, 1970.)

A. L. Gol'denveizer and A. V. Kolos (1965), 'On the Derivation of Two-Dimensional Equations in the Theory of Thin Elastic Plates', PMM, 29, 141-155.

A. V. Kolos (1964), 'On a Refinement of the Classical Theory of Bending of Circular Plates', PMM, 28, 582-589.

E. L. Reiss (1962), 'Symmetric Bending of Thick Circular Plates', J. Soc. Indust. Appl. Maths. 10, 596-609.

E. L. Reiss and S. Locke (1961), 'On the Theory of Plane Stress', Quart. Appl. Maths. 19, 195-203.

E. Reissner (1963), 'On the Derivation of Boundary Conditions for Plate Theory', Proc. Royal. Soc. $A, 276,178-186$.

M. I. Gusein-Zade (1965), 'On necessary and sufficient conditions for the existence of decaying solutions of the plane problem of the theory of elasticity for a semistrip', PMM 29, 892-901.

\section{Department of Mathematics and Computer Science}

Queensland Institute of Technology

Australia 